International Journal of Pure and Applied Mathematics

Volume 87 No. 3 2013, 459-474

ISSN: 1311-8080 (printed version); ISSN: 1314-3395 (on-line version)

url: http://www.ijpam.eu

doi: http://dx.doi.org/10.12732/ijpam.v87i3.10

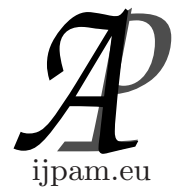

\title{
ROBUST EXPONENTIAL STABILITY OF LINEAR NEUTRAL SYSTEM WITH CONSTANT NEUTRAL AND TIME-VARYING DISCRETE DELAYS
}

\author{
Thaned Rojsiraphisal ${ }^{1 \S}$, Warakorn Sudsanguan ${ }^{2}$ \\ ${ }^{1}$ Department of Mathematics \\ Faculty of Science \\ Chiang Mai University \\ Chiang Mai, 50200, THAILAND \\ ${ }^{1} 239$ HuayKaew Rd., A. Muang \\ Chiang Mai, 50200, THAILAND \\ ${ }^{2}$ Department of Mathematics and Applied Statistics \\ Rajabhat Rajanagarindra University \\ Chachoengsao, 24000, THAILAND
}

\begin{abstract}
In this paper, an exponentially stable condition for linear neutral with uncertainties and constant neutral and time-varying discrete delays is formulated. Based on a change of variable and improved Lyapunov-Krasovskii functional, the sufficient condition is obtained in the form of linear matrix inequalities. Two numerical examples are given to show effectiveness of the proposed condition by comparing upper bounds of the time-delays and rate of convergence with recent works.
\end{abstract}

AMS Subject Classification: 93D09

Key Words: neutral system, uncertainty, exponential stability, linear matrix inequality

\section{Introduction}

In the past decades, the stability of neutral system has received a considerable

Received: July 9, 2013

(c) 2013 Academic Publications, Ltd.

$\S$ Correspondence author url: www.acadpubl.eu 
amount of attention from researchers ([1], [3], [4],[5], [8],[10], [11], [13],[14], [15], [16], [17], [18], [19],[20], [21] and the reference therein). Because the neutral has time-delays in both the state and its derivative (so-called discrete and neutral delays), it can be used to describe various practical applications in sciences and engineering such as aircraft stabilization, lossless transmission lines, population ecology, chemical processes, etc [1], [2]. It is well-known that time-delays often cause instability of systems or downgrade systems' performance. Thus researchers have proposed sufficient criteria for the systems to be stable. For more realistic, systems sometimes be disturbed by a small amount of uncertainties. Thus the problem of robust stability analysis has been widely investigated in [1],[2],[3],[4], [5],[8],[9], [10],[11],[12],[13], [15],[16], [17], [19], [21].

Most research have developed stability conditions for asymptotic stability. However, there are some need of fast speed of convergence in many applications. The exponential stability which offers faster speed of convergence than asymptotic stability has been focused by many researchers such as [1], [2], [4],[9], $[17]$.

To obtain exponential stability conditions of such systems, several techniques such as descriptor transformation, triple-integral termin Lyapunov-Krasovskii functional, matrix decomposition, or free-weighting matrices have been used in many works. However, these techniques lead to larger size of linear matrix inequality (LMI). In this paper, we use rather typical techniques base on an improved Lyapunov-Krasovskii functional, along with a change of variable, the Leibniz-Newton formula, and integral inequalities to guarantee (robust) exponential stability of two linear neutral systems with and without uncertainties and along with constant neutral delay and time-varying discrete delay. The proposed criteria are demonstrated their effectiveness in two numerical examples by comparing upper bounds of the time-delays and the rate of convergence with results in recent works.

\section{Preliminaries}

Throughout this article, $*$ represents the elements below the main diagonal of a symmetric matrix. The notation $A>0$ means $A$ is positive definite; $A^{T}$ denotes the transpose of the matrix $A$. $\lambda(A)$ are eigenvalues of $A ; \lambda_{\max }(A):=$ $\max \{\operatorname{Re}(\lambda): \lambda \in \lambda(A)\} . x_{t}:=\{x(t+s): s \in[-\tau, 0]\} ;\left\|x_{t}\right\|:=\sup _{s \in[-\tau, 0]} \| x(t+$ $s) \|$ and $\|\cdot\|$ denotes the Euclidean norm of given vector or matrix. $R^{n \times n}$ denotes real value matrix with dimension $n \times n$. 
Consider the neutral system

$$
\dot{x}(t)=(\bar{A}+\Delta A(t)) x(t)+(\bar{B}+\Delta B(t)) x(t-h(t))+\bar{C} \dot{x}(t-\tau),
$$

where $t>0, x(t) \in R^{n}$ is the state vector of the system. $\bar{A}, \bar{B}, \bar{C} \in R^{n \times n}$ are known constant matrices with $\|\bar{C}\|<1$. The neutral delays $\tau$ is a positive constant and discrete delay $h(t)$ is a continuously differentiable function satisfying

$$
0 \leq h_{1} \leq h(t) \leq h_{2} \text { and } \dot{h} \leq \mu<1 .
$$

We assume the initial condition is $x(t+\theta)=\phi(\theta), \forall \theta \in\left[-\max \left\{\tau, h_{2}\right\}, 0\right]$ and $\phi(\cdot)$ is a differentiable vector-valued initial function.

The time-varying structured uncertainties $\Delta A(t)$ and $\Delta B(t)$ are of the form

$$
[\Delta A(t), \Delta B(t)]=G F(t)\left[E_{1}, E_{2}\right],
$$

where $G, E_{1}, E_{2}$ are known matrices with appropriated dimensions, and the time-varying matrix $F(t)$ satisfies $F^{T}(t) F(t) \leq I, t \geq 0$.

The following definition and lemmas are well-known and to be used in the main result.

Definition 1. System (1) is said to be exponentially stable if there exist scalars $K>0$ and the rate of convergence $\alpha>0$ such that

$$
\|x(t, \phi)\| \leq K e^{-\alpha t}\|\phi\|, \forall t>0 .
$$

Lemma 2. [7] Consider the time-delay system

$$
\dot{x}(t)=f\left(t, x_{t}\right), x(t)=\phi(t), t \in[-h, 0] .
$$

If there exists a Lyapunov function $V\left(t, x_{t}\right)$ and $\lambda_{1}, \lambda_{2}>0$ such that for every solution $x(t)$ of the system, the following conditions hold:

$$
\begin{aligned}
& \text { (i) } \lambda_{1}\|x(t)\|^{2} \leq V\left(t, x_{t}\right) \leq \lambda_{2}\left\|x_{t}\right\|^{2}, \\
& \text { (ii) } \dot{V}\left(t, x_{t}\right) \leq 0
\end{aligned}
$$

then the solution of the system is bounded; i.e., there exists $N>0$ such that $\|x(t, \phi)\| \leq N\|\phi\|, \forall t \geq 0$.

Lemma 3. [12] Let $a(\gamma) \in R^{n}$ and $b(\gamma) \in R^{n}$ and $\gamma \in \Omega$. Then, for any symmetric positive definite matrix $X \in R^{n \times n}$ and any matrix $M \in R^{n \times n}$, the following holds:

$$
-2 \int_{\Omega} b^{T}(\gamma) a(\gamma) d \gamma \leq \int_{\Omega}\left[\begin{array}{l}
a(\gamma) \\
b(\gamma)
\end{array}\right]^{T} W\left[\begin{array}{l}
a(\gamma) \\
b(\gamma)
\end{array}\right] d \gamma
$$


where

$$
W=\left[\begin{array}{cc}
X & X M \\
M^{T} X & (X M+I)^{T} X^{-1}(X M+I)
\end{array}\right] .
$$

Lemma 4. [6] For any symmetric positive definite matrix $M \in \mathbb{R}^{n \times n}$, scalar $\sigma \geq 0$ and vector function $w:[0, \sigma] \rightarrow \mathbb{R}^{n}$ such that the integrations concerned are well defined, then

$$
\left(\int_{0}^{\sigma} w(s) d s\right)^{T} M\left(\int_{0}^{\sigma} w(s) d s\right) \leq \sigma \int_{0}^{\sigma} w^{T}(s) M w(s) d s .
$$

\section{Main Results}

Theorem 5. Let $0 \leq h_{1} \leq h(t) \leq h_{2}, \tau>0$ and $\eta=1 /(1-\mu)$. For given scalars $\alpha, \varepsilon>0$, the neutral system (1) is robustly exponentially stable with convergent rate $\alpha$ if there exist symmetric and positive-definite matrices $P, Q_{1}, Q_{2}, Q_{3}, N, W, R, U$ such that the following inequalities hold:

$$
\begin{aligned}
\Phi_{1} & =\Phi-\xi_{1}^{T} U \xi_{1}<0, \quad \xi_{1}=[0, I, 0,0,0,0,-I, 0]^{T}, \\
\text { and } \quad \Phi_{2} & =\Phi-\xi_{2}^{T} U \xi_{2}<0, \quad \xi_{2}=[0, I, 0,0,0,-I, 0,0]^{T},
\end{aligned}
$$

where

$$
\Phi=\left[\begin{array}{cccccccc}
Z_{11} & Z_{12} & Z_{13} & Z_{14} & Z_{15} & R & R & h_{2}(W+P)^{T} \\
* & Z_{22} & Z_{23} & Z_{24} & Z_{25} & U & U & 0 \\
* & * & Z_{33} & Z_{34} & Z_{35} & 0 & 0 & 0 \\
* & * & * & Z_{44} & Z_{45} & 0 & 0 & 0 \\
* & * & * & * & Z_{55} & 0 & 0 & 0 \\
* & * & * & * & * & -R-U & 0 & 0 \\
* & * & * & * & * & * & -R-U & 0 \\
* & * & * & * & * & * & * & -N
\end{array}\right]
$$

and

$$
\begin{aligned}
Z_{11}= & P\left(A+B_{2}\right)+\left(A+B_{2}\right)^{T} P+W B_{2}+B_{2}^{T} W+Q_{1}+Q_{2}+\eta\left(B_{2} A\right)^{T} N B_{2} A \\
& +A^{T} Q_{3} A+\left(h_{1}^{2}+h_{2}^{2}\right) A^{T} R A+\left(h_{2}-h_{1}\right)^{2} A^{T} U A-2 R+\varepsilon E_{1}^{T} E_{1}, \\
Z_{12}= & \eta\left(B_{2} A\right)^{T} N B_{2} B_{2}+A^{T} Q_{3} B_{2}-W B_{1}+\left(h_{1}^{2}+h_{2}^{2}\right) A^{T} R B_{2} \\
& +\left(h_{2}-h_{1}\right)^{2} A^{T} U B_{2}+\varepsilon e^{\alpha h_{2}} E_{1}^{T} E_{2}
\end{aligned}
$$




$$
\begin{aligned}
Z_{13}= & -\eta\left(B_{1} A\right)^{T} N B_{1} C-A^{T} Q_{3} C-P C-\left(h_{1}^{2}+h_{2}^{2}\right) A^{T} R C-\left(h_{2}-h_{1}\right)^{2} A^{T} U C, \\
Z_{14}= & \eta\left(B_{2} A\right)^{T} N B_{2} D+A^{T} Q_{3} D+P D+\left(h_{1}^{2}+h_{2}^{2}\right) A^{T} R D+\left(h_{2}-h_{1}\right)^{2} A^{T} U D, \\
Z_{15}= & \eta\left(B_{2} A\right)^{T} N B_{2} G+A^{T} Q_{3} G+P G+\left(h_{1}^{2}+h^{2}\right) A^{T} R G+\left(h_{2}-h_{1}\right)^{2} A^{T} U G, \\
Z_{22}= & \eta\left(B_{2} B_{2}\right)^{T} N B_{2} B_{2}+B_{2}^{T} Q_{3} B_{2}+\left(h_{1}^{2}+h_{2}^{2}\right) B_{2}^{T} R B_{2}+\left(h_{2}-h_{1}\right)^{2} B_{2}^{T} U B_{2} \\
& -(1-\mu) Q_{1}-2 U+\varepsilon e^{2 \alpha h_{2}} E_{2}^{T} E_{2} \\
Z_{23}= & -\eta\left(B_{1} B_{1}\right)^{T} N B_{1} C-B_{1}^{T} Q_{3} C-\left(h_{1}^{2}+h_{2}^{2}\right) B_{1}^{T} R C-\left(h_{2}-h_{1}\right)^{2} B_{1}^{T} U C \\
Z_{24}= & \eta\left(B_{2} B_{2}\right)^{T} N B_{2} D+B_{2}^{T} Q_{3} D+\left(h_{1}^{2}+h_{2}^{2}\right) B_{2}^{T} R D+\left(h_{2}-h_{1}\right)^{2} B_{2}^{T} U D \\
Z_{25}= & \eta\left(B_{2} B_{2}\right)^{T} N B_{2} G+B_{2}^{T} Q_{3} G+\left(h_{1}^{2}+h_{2}^{2}\right) B_{2}^{T} R G+\left(h_{2}-h_{1}\right)^{2} B_{2}^{T} U G \\
Z_{33}= & \eta\left(B_{2} C\right)^{T} N B_{2} C+C^{T} Q_{3} C+\left(h_{1}^{2}+h_{2}^{2}\right) C^{T} R C+\left(h_{2}-h_{1}\right)^{2} C^{T} U C-Q_{2} \\
Z_{34}= & -\eta\left(B_{1} C\right)^{T} N B_{1} D-C^{T} Q_{3} D-\left(h_{1}^{2}+h_{2}^{2}\right) C^{T} R D-\left(h_{2}-h_{1}\right)^{2} C^{T} U D \\
Z_{35}= & -\eta\left(B_{1} C\right)^{T} N B_{1} G-C^{T} Q_{3} G-\left(h_{1}^{2}+h_{2}^{2}\right) C^{T} R G-\left(h_{2}-h_{1}\right)^{2} C^{T} U G \\
Z_{44}= & \eta\left(B_{2} D\right)^{T} N B_{2} D+D^{T} Q_{3} D+\left(h_{1}^{2}+h_{2}^{2}\right) D^{T} R D+\left(h_{2}-h_{1}\right)^{2} D^{T} U D-Q_{3} \\
Z_{45}= & \eta\left(B_{2} D\right)^{T} N B_{2} G+D^{T} Q_{3} G+\left(h_{1}^{2}+h_{2}^{2}\right) D^{T} R G+\left(h_{2}-h_{1}\right)^{2} D^{T} U G \\
Z_{55}= & \eta\left(B_{2} G\right)^{T} N B_{2} G+G^{T} Q_{3} G+\left(h_{1}^{2}+h_{2}^{2}\right) G^{T} R G+\left(h_{2}-h_{1}\right)^{2} G^{T} U G-\varepsilon .
\end{aligned}
$$

Proof. We begin by changing variable

$$
z(t)=e^{\alpha t} x(t), \alpha>0 .
$$

With the uncertainties in Eq. (2), the neutral system in Eq. (1) becomes

$$
\dot{z}(t)=A z(t)+B z(t-h(t))-C z(t-\tau)+D \dot{z}(t-\tau)+G y(t),
$$

where $A=\bar{A}+\alpha I, B=e^{\alpha h(t)} \bar{B}, C=\alpha e^{\alpha \tau} \bar{C}, D=e^{\alpha \tau} \bar{C}$ and $y(t)=F(t)\left[E_{1} z(t)+\right.$ $\left.e^{\alpha h(t)} E_{2} z(t-h(t))\right]$.

Next consider a Lyapunov-Krasovskii functional candidate, $V\left(t, z_{t}\right)=V_{1}+V_{2}+V_{3}+V_{4}+V_{5}+V_{6}+V_{7}+V_{8}$ where

$$
\begin{aligned}
& V_{1}\left(z_{t}\right)=z^{T}(t) P z(t) \\
& V_{2}\left(z_{t}\right)=\frac{1}{1-\mu} \int_{t-h(t)}^{t}(h(t)-t+s) \dot{z}^{T}(s) B^{T} X B \dot{z}(s) d s d \beta \\
& V_{3}\left(z_{t}\right)=\int_{t-h(t)}^{t} z^{T}(s) Q_{1} z(s) d s, \quad V_{4}\left(z_{t}\right)=\int_{t-\tau}^{t} z^{T}(s) Q_{2} z(s) d s
\end{aligned}
$$




$$
\begin{aligned}
& V_{5}\left(z_{t}\right)=\int_{t-\tau}^{t} \dot{z}^{T}(s) Q_{3} \dot{z}(s) d s, \quad V_{6}\left(z_{t}\right)=h_{1} \int_{-h_{1}}^{0} \int_{t+\beta}^{t} \dot{z}^{T}(s) R \dot{z}(s) d s d \beta \\
& V_{7}\left(z_{t}\right)=h_{2} \int_{-h_{2}}^{0} \int_{t+\beta}^{t} \dot{z}^{T}(s) R \dot{z}(s) d s d \beta \\
& V_{8}\left(z_{t}\right)=\left(h_{2}-h_{1}\right) \int_{-h_{2}}^{-h_{1}} \int_{t+\beta}^{t} \dot{z}^{T}(s) U \dot{z}(s) d s d \beta .
\end{aligned}
$$

The derivatives of $V_{i}, i=1,2, \ldots, 8$ along the trajectory ( 7 ) are following:

$$
\dot{V}_{1}=2 z^{T}(t) P \dot{z}(t)
$$

With the Leibniz-Newton formula, Eq. (7) becomes

$$
\dot{z}(t)=(A+B) z(t)-B \int_{t-h(t)}^{t} \dot{z}(s) d s-C z(t-\tau)+D \dot{z}(t-\tau)+G y(t) .
$$

Then we have

$$
\dot{V}_{1}=2 z^{T}(t) P\left[(A+B) z(t)-B \int_{t-\tau}^{t} \dot{z}(s) d s+C \dot{z}(t-h)-D z(t-h)+G y(t)\right]
$$

Apply lemma 3 , with $a(\gamma)=B \dot{z}(s), b(\gamma)=P z(t)$ and the Leibniz-Newton formula, we obtain

$$
\begin{array}{r}
-2 \int_{t-h(t)}^{t} z^{T}(t) P B \dot{z}(s) d s \leq \int_{t-h(t)}^{t} \dot{z}^{T}(s) B^{T} X B \dot{z}(s) d s+2 z^{T}(t) P M^{T} X B \\
* \int_{t-h(t)}^{t} \dot{z}(s) d s+h_{2} z^{T}(t) P\left(M^{T} X+I\right) X^{-1}\left(M^{T} X+I\right) P z(t) .
\end{array}
$$

Let $W=P M^{T} X, N=h_{2} X$. We then obtain

$$
\begin{aligned}
\dot{V}_{1} & \leq z^{T}(t)[2 P(A+B) z(t)+2 W B z(t)-2 W B z(t-h(t)) \\
& +h_{2}^{2}(W+P) N^{-1}(W+P)^{T} z(t)-2 P C \dot{z}(t-\tau) \\
& +2 P D \dot{z}(t-\tau)+2 P G y(t)]+\int_{t-h(t)}^{t} \dot{z}^{T}(s) B^{T} X B \dot{z}(s) d s \\
\dot{V}_{2} & =\frac{h(t)}{1-\mu} \dot{z}^{T}(t) B^{T} X B \dot{z}(t)-\frac{1-h(t)}{1-\mu} \int_{t-h(t)}^{t} \dot{z}^{T}(s) B^{T} X B \dot{z}(s) d s \\
& \leq \frac{h_{2}}{1-\mu} \dot{z}^{T}(t) B^{T} X B \dot{z}(t)-\int_{t-h(t)}^{t} \dot{z}^{T}(s) B^{T} X B \dot{z}(s) d s
\end{aligned}
$$




$$
\begin{aligned}
= & \frac{h_{2}}{1-\mu}[A z(t)+B z(t-h(t))-C z(t-\tau)+D \dot{z}(t-\tau)+G y(t)]^{T} \\
& * B^{T} X B[A z(t)+B z(t-h(t))-C z(t-\tau)+D \dot{z}(t-\tau)+G y(t)] \\
& -\int_{t-h(t)}^{t} \dot{z}^{T}(s) B^{T} X B \dot{z}(s) d s \\
\dot{V}_{3} \leq & z^{T}(t) Q_{1} z(t)-(1-\mu) z^{T}(t-h(t)) Q_{1} z(t-h(t)) \\
\dot{V}_{4}= & z^{T}(t) Q_{2} z(t)-z^{T}(t-\tau) Q_{2} z(t-\tau) \\
\dot{V}_{5}= & \dot{z}^{T}(t) Q_{3} \dot{z}(t)-\dot{z}^{T}(t-\tau) Q_{3} \dot{z}(t-\tau) \\
& \dot{V}_{6}=h_{1}^{2} \dot{z}^{T}(t) R \dot{z}(t)-h_{1} \int_{t-h_{1}}^{t} \dot{z}^{T}(s) R \dot{z}(s) d s \\
& \dot{V}_{7}=h_{2}^{2} \dot{z}^{T}(t) R \dot{z}(t)-h_{2} \int_{t-h_{2}}^{t} \dot{z}^{T}(s) R \dot{z}(s) d s \\
& \dot{V}_{8}=\left(h_{2}-h_{1}\right)^{2} \dot{z}^{T}(t) U \dot{z}(t)-\left(h_{2}-h_{1}\right) \int_{t-h_{2}}^{t-h_{1}} \dot{z}^{T}(s) U \dot{z}(s) d s
\end{aligned}
$$

Apply lemma 4 and the Newton-Leibniz formula to $\dot{V}_{6}$ and $\dot{V}_{7}$, we have

$$
\begin{aligned}
\dot{V}_{6} \leq h_{1}^{2} \dot{z}^{T}(t) R \dot{z}(t)-z^{T}(t) R z(t)+2 z^{T}(t) R z( & \left.-h_{1}\right) \\
& -z^{T}\left(t-h_{1}\right) R z\left(t-h_{1}\right), \\
\dot{V}_{7} \leq h_{2}^{2} \dot{z}^{T}(t) R \dot{z}(t)-z^{T}(t) R z(t)+2 z^{T}(t) R z(t & \left.-h_{2}\right) \\
& -z^{T}\left(t-h_{2}\right) R z\left(t-h_{2}\right) .
\end{aligned}
$$

Since $h(t)$ is bounded continuous function, we have

$$
\begin{aligned}
\dot{V}_{8} & =\left(h_{2}-h_{1}\right)^{2} \dot{z}^{T}(t) U \dot{z}(t)-\left(h_{2}-h(t)\right) \int_{t-h_{2}}^{t-h(t)} \dot{z}^{T}(s) U \dot{z}(s) d s \\
& -\left(h(t)-h_{1}\right) \int_{t-h_{2}}^{t-h(t)} \dot{z}^{T}(s) U \dot{z}(s) d s-\left(h(t)-h_{1}\right) \int_{t-h(t)}^{t-h_{1}} \dot{z}^{T}(s) U \dot{z}(s) d s \\
& -\left(h_{2}-h(t)\right) \int_{t-h(t)}^{t-h_{1}} \dot{z}^{T}(s) U \dot{z}(s) d s .
\end{aligned}
$$

Let $\beta=\frac{h_{2}-h(t)}{h_{2}-h_{1}} \leq 1$. Thus, we have

$$
-\left(h(t)-h_{1}\right) \int_{t-h_{2}}^{t-h(t)} \dot{z}^{T}(s) U \dot{z}(s) d s=-(1-\beta) \int_{t-h_{2}}^{t-h(t)} \dot{z}^{T}(s) U \dot{z}(s) d s
$$


$-\left(h_{2}-h(t)\right) \int_{t-h(t)}^{t-h_{1}} \dot{z}^{T}(s) U \dot{z}(s) d s=-\beta\left(h_{2}-h_{1}\right) \int_{t-h(t)}^{t-h_{1}} \dot{z}^{T}(s) U \dot{z}(s) d s$.

Applying lemma 4 and the Newton-Leibniz formula, we obtain

$$
\begin{aligned}
\dot{V}_{8} & \leq\left(h_{2}-h_{1}\right)^{2} \dot{z}^{T}(t) U \dot{z}(t)-z^{T}(t-h(t)) U z(t-h(t)) \\
& +2 z^{T}(t-h(t)) U z\left(t-h_{1}\right) \\
& +2 z^{T}(t-h(t)) U z\left(t-h_{2}\right)-2 z^{T}\left(t-h_{2}\right) U z\left(t-h_{2}\right) \\
& -2 z^{T}\left(t-h_{1}\right) U z\left(t-h_{1}\right)+\beta\left[2 z^{T}\left(t-h_{1}\right) U z(t-h(t))\right. \\
& \left.-z^{T}\left(t-h_{1}\right) U z\left(t-h_{1}\right)-z^{T}(t-h(t)) U z(t-h(t))\right] \\
& +(1-\beta)\left[2 z^{T}\left(t-h_{2}\right) U z(t-h(t))\right. \\
& \left.-z^{T}\left(t-h_{2}\right) U z\left(t-h_{2}\right)-z^{T}(t-h(t)) U z(t-h(t))\right] .
\end{aligned}
$$

With the uncertainties as in Eq. (2), it follows that for any $\varepsilon>0$, we have

$$
\varepsilon\left[E_{1} z(t)+e^{\alpha h(t)} E_{2} z(t-h(t))\right]^{T}\left[E_{1} z(t)+e^{\alpha \tau} E_{2} z(t-h(t))\right] \geq \varepsilon y^{T}(t) y(t) .
$$

Since $B=e^{\alpha h(t)} \bar{B}, h_{1} \leq h(t) \leq h_{2}$, we define $B_{1}=e^{\alpha h_{1}} \bar{B}$ and $B_{2}=e^{\alpha h_{2}} \bar{B}$. Combining Eqs. (8) - (16), we obtain

$$
\begin{aligned}
\dot{V} \leq & z^{T}(t)\left[2 P\left(A+B_{2}\right)+2 W B_{2}+\eta\left(B_{2} A\right)^{T} N B_{2} A+h_{2}^{2}(W+P) N^{-1}(W+P)^{T}\right. \\
& +A^{T} Q_{3} A+\left(h_{1}^{2}+h_{2}^{2}\right) A^{T} R A+\left(h_{2}-h_{1}\right)^{2} A^{T} U A-2 R+Q_{1}+Q_{2} \\
& \left.+\varepsilon E_{1}^{T} E_{1}\right] z(t) \\
& +z^{T}(t)\left[2 \eta\left(B_{2} A\right)^{T} N B_{2} B_{2}+2 A^{T} Q_{3} B_{2}-2 W B_{1}+2\left(h_{1}^{2}+h_{2}^{2}\right) A^{T} R B_{2}\right. \\
& \left.+2\left(h_{2}-h_{1}\right)^{2} A^{T} U B_{2}+2 \varepsilon e^{\alpha h_{2}} E_{1}^{T} E_{2}\right] z(t-h(t)) \\
& +z^{T}(t)\left[-2 \eta\left(B_{1} A\right)^{T} N B_{1} C\right. \\
& \left.-2 A^{T} Q_{3} C-2 P C-2\left(h_{1}^{2}+h_{2}^{2}\right) A^{T} R C-2\left(h_{2}-h_{1}\right)^{2} A^{T} U C\right] z(t-\tau) \\
& +z^{T}(t)\left[2 \eta\left(B_{2} A\right)^{T} N B_{2} D+2 A^{T} Q_{3} D+2 P D+2\left(h_{1}^{2}+h_{2}^{2}\right) A^{T} R D\right. \\
& \left.+2\left(h_{2}-h_{1}\right)^{2} A^{T} U D\right] \dot{z}(t-\tau)+z^{T}(t)\left[2 \eta\left(B_{2} A\right)^{T} N B_{2} G+2 A^{T} Q_{3} G+2 P G\right.
\end{aligned}
$$




$$
\begin{aligned}
& \left.+2\left(h_{1}^{2}+h^{2}\right) A^{T} R G+2\left(h_{2}-h_{1}\right)^{2} A^{T} U G\right] y(t) \\
& +z^{T}(t)\left[2 R z\left(t-h_{1}\right)+2 R z\left(t-h_{2}\right)\right] \\
& +z^{T}(t-h(t))\left[\eta\left(B_{2} B_{2}\right)^{T} N B_{2} B_{2}+B_{2}^{T} Q_{3} B_{2}+\left(h_{1}^{2}+h_{2}^{2}\right) B_{2}^{T} R B_{2}\right. \\
& \left.+\left(h_{2}-h_{1}\right)^{2} B_{2}^{T} U B_{2}-(1-\mu) Q_{1}-2 U+\varepsilon e^{2 \alpha h_{2}} E_{2}^{T} E_{2}\right] z(t-h(t)) \\
& +z^{T}(t-h(t))\left[-2 \eta\left(B_{1} B_{1}\right)^{T} N B_{1} C-2 B_{1}^{T} Q_{3} C-2\left(h_{1}^{2}+h_{2}^{2}\right) B_{1}^{T} R C\right. \\
& \left.-2\left(h_{2}-h_{1}\right)^{2} B_{1}^{T} U C\right] z(t-\tau)+z^{T}(t-h(t))\left[2 \eta\left(B_{2} B_{2}\right)^{T} N B_{2} D+2 B_{2}^{T} Q_{3} D\right. \\
& \left.+2\left(h_{1}^{2}+h_{2}^{2}\right) B_{2}^{T} R D+2\left(h_{2}-h_{1}\right)^{2} B_{2}^{T} U D\right] \dot{z}(t-\tau)+z^{T}(t-h(t))\left[2 B_{2}^{T} Q_{3} G\right. \\
& +2 \eta\left(B_{2} B_{2}\right)^{T} N B_{2} G+2 B_{2}^{T} Q_{3} G+2\left(h_{1}^{2}+h_{2}^{2}\right) B_{2}^{T} R G \\
& \left.+2\left(h_{2}-h_{1}\right)^{2} B_{2}^{T} U G\right] y(t) \\
& +z^{T}(t-h(t))\left[2 U z\left(t-h_{1}\right)+2 U z\left(t-h_{2}\right)\right] \\
& +z^{T}(t-\tau)\left[\eta\left(B_{2} C\right)^{T} N B_{2} C+C^{T} Q_{3} C\right. \\
& \left.+\left(h_{1}^{2}+h_{2}^{2}\right) C^{T} R C+\left(h_{2}-h_{1}\right)^{2} C^{T} U C-Q_{2}\right] z(t-\tau) \\
& +z^{T}(t-\tau)\left[-2 C^{T} Q_{3} D\right. \\
& \left.-2 \eta\left(B_{1} C\right)^{T} N B_{1} D-2\left(h_{1}^{2}+h_{2}^{2}\right) C^{T} R D-2\left(h_{2}-h_{1}\right)^{2} C^{T} U D\right] \dot{z}(t-\tau) \\
& +z^{T}(t-\tau)\left[-2 \eta\left(B_{1} C\right)^{T} N B_{1} G-2 C^{T} Q_{3} G-2\left(h_{1}^{2}+h_{2}^{2}\right) C^{T} R G\right. \\
& \left.-2\left(h_{2}-h_{1}\right)^{2} C^{T} U G\right] y(t)+\dot{z}^{T}(t-\tau)\left[\eta\left(B_{2} D\right)^{T} N B_{2} D+D^{T} Q_{3} D\right. \\
& \left.+\left(h_{1}^{2}+h_{2}^{2}\right) D^{T} R D+\left(h_{2}-h_{1}\right)^{2} D^{T} U D-Q_{3}\right] \dot{z}(t-\tau) \\
& +\dot{z}^{T}(t-\tau)\left[2 \eta\left(B_{2} D\right)^{T} N B_{2} G+2 D^{T} Q_{3} G+2\left(h_{1}^{2}+h_{2}^{2}\right) D^{T} R G\right. \\
& \left.+2\left(h_{2}-h_{1}\right)^{2} D^{T} U G\right] y(t)+y^{T}(t)\left[\eta\left(B_{2} G\right)^{T} N B_{2} G+G^{T} Q_{3} G\right. \\
& \left.+\left(h_{1}^{2}+h_{2}^{2}\right) G^{T} R G+\left(h_{2}-h_{1}\right)^{2} G^{T} U G-\varepsilon\right] y(t) \\
& +z^{T}\left(t-h_{1}\right)[-R-U] z\left(t-h_{1}\right)+z^{T}\left(t-h_{2}\right)[-R-U] z\left(t-h_{2}\right) \\
& -\beta\left[z\left(t-h_{1}\right)-z(t-h(t))\right]^{T} U\left[z\left(t-h_{1}\right)-z(t-h(t))\right] \\
& -(1-\beta)\left[z(t-h(t))-z\left(t-h_{2}\right)\right]^{T} U\left[z(t-h(t))-z\left(t-h_{2}\right)\right]
\end{aligned}
$$




$$
\begin{aligned}
= & \Omega^{T} \Phi^{*} \Omega-\beta\left[z\left(t-h_{1}\right)-z(t-h(t))\right]^{T} U\left[z\left(t-h_{1}\right)-z(t-h(t))\right] \\
& -(1-\beta)\left[z(t-h(t))-z\left(t-h_{2}\right)\right]^{T} U\left[z(t-h(t))-z\left(t-h_{2}\right)\right],
\end{aligned}
$$

where $\Omega=\left[z(t), z(t-h(t)), z(t-\tau), \dot{z}(t-\tau), y(t), z\left(t-h_{1}\right), z\left(t-h_{2}\right)\right]^{T}$ and

$$
\Phi^{*}=\left[\begin{array}{ccccccc}
Z_{11}^{*} & Z_{12} & Z_{13} & Z_{14} & Z_{15} & R & R \\
* & Z_{22} & Z_{23} & Z_{24} & Z_{25} & U & U \\
* & * & Z_{33} & Z_{34} & Z_{35} & 0 & 0 \\
* & * & * & Z_{44} & Z_{45} & 0 & 0 \\
* & * & * & * & Z_{55} & 0 & 0 \\
* & * & * & * & * & -R-U & 0 \\
* & * & * & * & * & * & -R-U
\end{array}\right]
$$

where $Z_{11}^{*}=2 P\left(A+B_{2}\right)+2 W B_{2}+\eta\left(B_{2} A\right)^{T} N B_{2} A-2 R+Q_{1}+Q_{2}+A^{T} Q_{3} A$ $+\left(h_{1}^{2}+h_{2}^{2}\right) A^{T} R A+\left(h_{2}-h_{1}\right)^{2} A^{T} U A+\varepsilon E_{1}^{T} E_{1}+h_{2}^{2}(W+P) N^{-1}(W+P)^{T}$.

Define $\quad \Phi_{1}^{*}=\Phi^{*}-[0, I, 0,0,0,0,-I]^{T} U[0, I, 0,0,0,0,-I]$

and

$$
\Phi_{2}^{*}=\Phi^{*}-[0, I, 0,0,0,-I, 0]^{T} U[0, I, 0,0,0,-I, 0] .
$$

Therefore, the derivative of $V(t)$ is bounded above by

$$
\Omega^{T}\left[(1-\beta) \Phi_{1}^{*}+\beta \Phi_{2}^{*}\right] \Omega .
$$

Since $0 \leq \beta \leq 1$ and $(1-\beta) \Phi_{1}^{*}+\beta \Phi_{2}^{*}$ is a convex combination between $\Phi_{1}^{*}$ and $\Phi_{2}^{*}$, thus, if $(1-\beta) \Phi_{1}^{*}+\beta \Phi_{2}^{*}<0$, it is equivalent to $\Phi_{1}^{*}<0$ and $\Phi_{2}^{*}<0$. This implies that $\dot{V}<0$. Since $\Phi^{*}$ is nonlinear matrix equality, thus, we apply Schur compliment to it, one can see that the non-linear matrix $\Phi^{*}$ is equivalent to the linear matrix $\Phi$. It follows that the Since $\Phi_{1}<0$ and $\Phi_{2}<0$.

With the Lyapunov-Krasovskii functionals defined above, we have

$$
\lambda_{1}\|z(t)\|^{2} \leq V\left(t, z_{t}\right) \leq \lambda_{2}\left\|z_{t}\right\|^{2},
$$

where $\lambda_{1}=\lambda_{\min }(P)$ and $\lambda_{2}=\lambda_{\max }(P)+\frac{h_{2}^{2}}{1-\mu} \lambda_{\max }\left(B^{T} X B\right)+h_{2} \lambda_{\max }\left(Q_{1}\right)+$ $\tau \lambda_{\max }\left(Q_{2}\right)+\tau \lambda_{\max }\left(Q_{3}\right)+\left(h_{1}^{3}+h_{2}^{3}\right) \lambda_{\max }(R)+\left(h_{2}-h_{1}\right)^{3} \lambda_{\max }(U)$. By lemma 2 , there exists $K_{1}>0$ such that $\|z(t, \phi)\| \leq K_{1}\|\phi\|, \forall t \geq 0$. With the change of variable as in Eq. (6), this implies that the solution $x(t, \phi)$ satisfies $\|x(t, \phi)\| \leq$ $K\|\phi\| e^{-\alpha t}, \forall t \geq 0$. Thus, the robust exponentially stability criterion of Eq. (1) is obtained. The proof is complete. 
Next we consider the linear neutral system without uncertainties and mixed delays of the form:

$$
\dot{x}(t)=\bar{A} x(t)+\bar{B} x(t-h(t))+\bar{C} \dot{x}(t-\tau) .
$$

The exponential stability of the linear neutral with mixed delays (19) can be stated in the following corollary.

Corollary 6. Let $\tau>0,0 \leq h_{1} \leq h(t) \leq h_{2}$, and $\dot{h} \leq \mu<1, \eta=$ $1 /(1-\mu)$. For given scalars $\alpha>0$, the neutral system (19) is robustly exponentially stable with convergent rate $\alpha$ if there exist symmetric and positivedefinite matrices $P, Q_{1}, Q_{2}, Q_{3}, N, W, R, U$ such that the following inequality holds:

$$
\begin{aligned}
\Pi_{1} & =\Pi-\omega_{1}^{T} U \omega_{1}<0, \quad \omega_{1}=[0, I, 0,0,0,-I, 0]^{T}, \\
\text { and } \quad \Pi_{2} & =\Pi-\omega_{2}^{T} U \omega_{2}<0, \quad \omega_{2}=[0, I, 0,0,-I, 0,0]^{T},
\end{aligned}
$$

where

$$
\Pi=\left[\begin{array}{ccccccc}
Y_{11} & Y_{12} & Y_{13} & Y_{14} & R & R & h_{2}(W+P)^{T} \\
* & Y_{22} & Y_{23} & Y_{24} & U & U & 0 \\
* & * & Y_{33} & Y_{34} & 0 & 0 & 0 \\
* & * & * & Y_{44} & 0 & 0 & 0 \\
* & * & * & * & -R-U & 0 & 0 \\
* & * & * & * & * & -R-U & 0 \\
* & * & * & * & * & * & -N
\end{array}\right]
$$

and

$$
\begin{aligned}
Y_{11}= & P\left(A+B_{2}\right)+\left(A+B_{2}\right)^{T} P+W B_{2}+B_{2}^{T} W+Q_{1}+Q_{2}+\eta\left(B_{2} A\right)^{T} N B_{2} A \\
& +A^{T} Q_{3} A+\left(h_{1}^{2}+h_{2}^{2}\right) A^{T} R A+\left(h_{2}-h_{1}\right)^{2} A^{T} U A-2 R, \\
Y_{12}= & \eta\left(B_{2} A\right)^{T} N B_{2} B_{2}+A^{T} Q_{3} B_{2}-W B_{1}+\left(h_{1}^{2}+h_{2}^{2}\right) A^{T} R B_{2} \\
& +\left(h_{2}-h_{1}\right)^{2} A^{T} U B_{2}, \\
Y_{13}= & -\eta\left(B_{1} A\right)^{T} N B_{1} C-A^{T} Q_{3} C-P C-\left(h_{1}^{2}+h_{2}^{2}\right) A^{T} R C-\left(h_{2}-h_{1}\right)^{2} A^{T} U C, \\
Y_{14}= & \eta\left(B_{2} A\right)^{T} N B_{2} D+A^{T} Q_{3} D+P D+\left(h_{1}^{2}+h_{2}^{2}\right) A^{T} R D+\left(h_{2}-h_{1}\right)^{2} A^{T} U D, \\
Y_{22}= & \eta\left(B_{2} B_{2}\right)^{T} N B_{2} B_{2}+B_{2}^{T} Q_{3} B_{2}+\left(h_{1}^{2}+h_{2}^{2}\right) B_{2}^{T} R B_{2}+\left(h_{2}-h_{1}\right)^{2} B_{2}^{T} U B_{2} \\
& -(1-\mu) Q_{1}-2 U, \\
Y_{23}= & -\eta\left(B_{1} B_{1}\right)^{T} N B_{1} C-B_{1}^{T} Q_{3} C-\left(h_{1}^{2}+h_{2}^{2}\right) B_{1}^{T} R C-\left(h_{2}-h_{1}\right)^{2} B_{1}^{T} U C \\
Y_{24}= & \eta\left(B_{2} B_{2}\right)^{T} N B_{2} D+B_{2}^{T} Q_{3} D+\left(h_{1}^{2}+h_{2}^{2}\right) B_{2}^{T} R D+\left(h_{2}-h_{1}\right)^{2} B_{2}^{T} U D
\end{aligned}
$$




$$
\begin{aligned}
& Y_{33}=\eta\left(B_{2} C\right)^{T} N B_{2} C+C^{T} Q_{3} C+\left(h_{1}^{2}+h_{2}^{2}\right) C^{T} R C+\left(h_{2}-h_{1}\right)^{2} C^{T} U C-Q_{2} \\
& Y_{34}=-\eta\left(B_{1} C\right)^{T} N B_{1} D-C^{T} Q_{3} D-\left(h_{1}^{2}+h_{2}^{2}\right) C^{T} R D-\left(h_{2}-h_{1}\right)^{2} C^{T} U D \\
& Y_{44}=\eta\left(B_{2} D\right)^{T} N B_{2} D+D^{T} Q_{3} D+\left(h_{1}^{2}+h_{2}^{2}\right) D^{T} R D+\left(h_{2}-h_{1}\right)^{2} D^{T} U D-Q_{3} .
\end{aligned}
$$

Proof. To obtain the exponential stability criterion for (19), we follow the proof of the theorem 5 with the same Lyapunov-Krasovskii functionals and assume $G=E_{1}=E_{2}=0, \varepsilon=0$. Then the proof is complete.

Remark 1. Both proposed exponential criteria with rate of convergence $\alpha$ are based on a change of variable and rather typical Lyapunov-Krasovskii functional except in $V_{2}$ that is chosen to complement the integral term arisen from $\dot{V}_{1}$. No free weight matrix are required. This keeps the size of LMI rather small comparing to other works.

Remark 2. In case of $\alpha=0$, one can easily see that the zero solutions of (1) and (19) are asymptotically stable.

Remark 3. Prior to check the feasibility of our criteria, one require the information of the matrices $A, B, C$ and $D$ which can be obtained by transforming as follow: $A=\bar{A}+\alpha I, B=e^{\alpha h(t)} \bar{B}, C=\alpha e^{\alpha \tau} \bar{C}$, and $D=e^{\alpha \tau} \bar{C}$.

\section{Numerical Examples}

To demonstrate the effectiveness of our proposed criteria, we compare the upper bounds on time-delays and convergent rate with other works. Here we solve the LMIs (3), (4), (20) and (21) using LMI control toolbox in MATLAB.

Example 1. Consider the linear neutral system (1) with uncertainties described in [10], [15], [16], [21] with

$$
\begin{aligned}
& \bar{A}=\left[\begin{array}{cc}
-2 & 0 \\
0 & -1
\end{array}\right], \quad \bar{B}=\left[\begin{array}{cc}
-1 & 0 \\
-1 & -1
\end{array}\right], \quad \bar{C}=\left[\begin{array}{ll}
c & 0 \\
0 & c
\end{array}\right], 0 \leq|c|<1, \\
& G=\left[\begin{array}{cc}
0.01 & 0 \\
0 & 0.04
\end{array}\right], E_{1}=\left[\begin{array}{cc}
\delta_{1} & 0 \\
0 & \delta_{2}
\end{array}\right], E_{2}\left[\begin{array}{cc}
\delta_{3} & 0 \\
0 & \delta_{4}
\end{array}\right] \\
& \left|\delta_{1}\right| \leq 1.6, \quad\left|\delta_{2}\right| \leq 0.05, \quad\left|\delta_{3}\right| \leq 0.1, \quad\left|\delta_{4}\right| \leq 0.3
\end{aligned}
$$

Clearly that $\|\bar{C}\|=c<1$ for all $c$. Setting $\mu=0.1, \delta_{1}=-0.1, \delta_{2}=-0.05, \delta_{3}=$ $0.01, \delta_{4}=0.01$ and solving the LMIs (3) and (4) from Theorem 5 for different 
values $c$ provides the upper bounds of discrete time delays $\left(h_{2}\right)$ (see Table ??). Note that the upper bounds provided in [10] are the same as those in [15] while the upper bounds $h_{2}$ obtained in Theorem 5 are larger than the others for $c \geq 0.2$ (in case of asymptotic stability, A.S.). We further investigate the convergent rates for the same upper bounds $h_{2}$ as given in [16] for $c \geq 0.2$ (see Table ??). This shows that for our criterion provides faster convergence. Finally, we list the upper bounded delays $h_{2}$ for various rate of changes of delay $\mu$ as in Table ??. Results show that the upper bounds decrease as $\mu$ increases.

\begin{tabular}{|c|c|c|c|c|c|c|c|c|}
\hline$c$ & 0 & 0.1 & 0.2 & 0.3 & 0.4 & 0.5 & 0.6 & 0.7 \\
\hline \multicolumn{8}{|c|}{ case asymptotically stable $(\alpha=0)$} \\
\hline Yu \& Lien[21] & 1.166 & 0.962 & 0.778 & 0.616 & 0.472 & 0.346 & 0.235 & 0.130 \\
Kwon et al. [10] & 1.107 & 0.920 & 0.751 & 0.599 & 0.462 & 0.340 & 0.231 & 0.127 \\
Qiu et al. [15] & 1.107 & 0.920 & 0.751 & 0.599 & 0.462 & 0.340 & 0.231 & 0.127 \\
Ramakrishnan \& Ray [16] ${ }^{*}$ & 1.177 & 0.971 & 0.786 & 0.622 & 0.478 & 0.349 & 0.235 & 0.130 \\
Thm 5 & 0.922 & 0.911 & 0.881 & 0.832 & 0.760 & 0.663 & 0.537 & 0.372 \\
\hline \hline convergent rate $\alpha$ for given upper bounds $h_{2}$ as in $[16]^{*}$ and $\tau=0.51$ \\
\hline Thm 5 & 0.118 & 0.215 & 0.273 & 0.306 & 0.323 & 0.330 \\
\hline
\end{tabular}

Table 1: Upper bounds $h_{2}$ with $h_{1}=0, \mu=0.1$

\begin{tabular}{|c|c|c|c|c|c|}
\hline$\mu$ & 0.1 & 0.3 & 0.5 & 0.7 & 0.9 \\
\hline Yu \& Lien[21] & 0.962 & 0.907 & 0.850 & 0.789 & 0.714 \\
Kwon et al. [10], Qiu et al. [15] & 0.920 & 0.862 & 0.804 & 0.753 & 0.726 \\
Ramakrishnan \& Ray [16] & 0.971 & 0.960 & 0.960 & 0.960 & 0.960 \\
Thm 5 & 0.911 & 0.750 & 0.572 & 0.371 & 0.135 \\
\hline
\end{tabular}

Table 2: Upper bounds delay $h_{2}$ for different $\mu$ and $h_{1}=0, c=0.1$

Example 2. Consider the linear neutral system without uncertainties (19) studied in [5], [13], [14], [19] with

$$
\bar{A}=\left[\begin{array}{cc}
-0.9 & 0.2 \\
0.1 & -0.9
\end{array}\right], \bar{B}=\left[\begin{array}{cc}
-1.1 & -0.2 \\
-0.1 & -1.1
\end{array}\right], \bar{C}=\left[\begin{array}{cc}
-0.2 & 0 \\
0.2 & -0.1
\end{array}\right] .
$$

Here $\|\bar{C}\|=0.292<1$. We first consider stability in the case of $h(t)$ is a constant i.e. $h(t)=h_{1}=h_{2}$ and $\tau=h$. Solving LMIs (20) and (21) in Corollary 6, the upper bounds $h$ are listed in Table ??. Our criteria provide the upper bound of $h=0.803$ (for A.S.) which is smaller than other works. However, the criteria from [5], [13], [14], [19] are only asymptotically stable but our criteria are guaranteeing exponentially stable (E.S.). Thus, we further list the upper bounds $h$ for E.S. case. Moreover, we continue investigate its stability for the case of $h(t)=h_{1}=h_{2}$ is a constant but $\tau \neq h$. With the 
various values of delays $h$ and $\tau$, the upper bounds of the convergent rates are list in Table ??. Results show that the convergent rate decreases as one delay fixed and the other delay increases.

\begin{tabular}{|c|c|c|c|c|c|}
\hline \multicolumn{7}{|c|}{ case $h(t)=h_{1}=h_{2}=\tau$} \\
\hline$\alpha$ & 0 & 0.1 & 0.7 & 1.3 & 1.7 \\
\hline Chen et al. [5] & 1.04 & - & - & - & - \\
Qian et al. [13] & 1.72 & - & - & - & - \\
Qian et al. [14] & 0.7 & - & - & - & - \\
Tian et al. [19] & 3.99 & - & - & - & - \\
Corollary 6 & 0.803 & 0.742 & 0.544 & 0.384 & 0.200 \\
\hline
\end{tabular}

Table 3: Upper bounds on time-delay with various $\alpha$ in Example 2

\begin{tabular}{|c|c|c|c|c|c|c|}
\hline$\tau$ & 0.1 & 0.5 & 1 & 1.5 & 2 & 2.5 \\
\hline$h=0.1$ & 1.8 & 1.43 & 1.116 & 0.873 & 0.701 & 0.579 \\
\hline$h=0.5$ & 0.687 & 0.601 & 0.507 & 0.431 & 0.373 & 0.327 \\
\hline$h=0.8$ & 0.004 & 0.004 & 0.004 & 0.004 & 0.004 & 0.004 \\
\hline
\end{tabular}

Table 4: Upper bounds of convergent rate $\alpha$ for various $h \neq \tau$

\section{Acknowledgments}

The first author thanks to Chiang Mai University for fully support of this work.

\section{References}

[1] I. Amri, D. Soudani, M. Benrejeb, On robust $\alpha$-stability analysis of uncertain neutral system with time varying delays: a novel augmented Lyapunov functional approach, the 8th International Multi-Conference on Systems, Signals and Devices, (2011), 1-6.

[2] T. Botmart, P. Niamsup, V.N. Pha, Delay-dependent exponential stabilization for uncertain linear systems with interval non-differentiable timevarying delays, Applied Mathematics and Computation, 217, No. 21 (2011), 8236-8247.

[3] W. Chen, W. Zheng, Delay-dependent robust stabilization for uncertain neutral systems with distributed delays, Automatica, 43, No.1 (2007), 95-104. 
[4] Y. Chen, A. Xue, R. Lu, S. Zhou, On robustly exponential stability of uncertain neutral systems with time-varying delays and nonlinear perturbation, Nonlinear Analysis, 68, No. 8 (2008), 2464-2470.

[5] H. Chen, Y. Zhang, Y. Zhao, Stability analysis for uncertain neutral systems with discrete and distributed delays, Applied Mathematics and Computation, 218, No. 23 (2012), 11351-11361.

[6] K. Gu, An integral inequality in the stability problem of time-delay systems, Proceedings of 39th IEEE Cdc Sydney, Australia, (2000), 2805-2801.

[7] K. Gu, V.L. Kharitonov, J. Chen, Stability of time-delay systems, Birkhauser, Berlin, (2003).

[8] F. E. Haoussi, E.H.Tissir, Delay and its time-derivative dependent robust stability of uncertain neutral systems with saturating actuators, International Journal of Automation and Computing, 7, No. 4 (2010), 455-462.

[9] O.M. Kwon, J.H. Park, Exponential stability of uncertain dynamic systems including state delay, Applied Mathematics Letters, 19, No. 9 (2006), 901907.

[10] O.M. Kwon, J.H. Park, and S.M. Lee, On delay-dependent robust stability of uncertain neutral systems with time-varying delay, Applied Mathematics Computation, 203, No. 2 (2008), 843-853.

[11] S. Lakshmanan, T. Senthilkumar, P. Balasubramaniam, Improved results on robust stability of neutral systems with mixed time-varying delays and nonlinear perturbations, Applied Mathematical Modelling, 35, No. 11 (2011), 5355-5368.

[12] P. Park, A delay-dependent stability criterion for systems with uncertain time-invariant delays, IEEE Transactions on Automatic Control, 44, No. 4 (1999), 876-877.

[13] W. Qian, J. Liu, Y. Sun, S. Fei, A less conservative robust stability criteria for uncertain neutral systems with mixed delays, Mathematics and Computers in Simulation, 80, No. 5 (2010), 1007-1017.

[14] W. Qian, J. Liu, S. Fei, Augmented Lyapunov functional approach for stability of neutral systems with mixed delays, Asian Journal of Control, 14, No. 2 (2012), 572-579. 
[15] F. Qiu, B. Cui and Y. Ji, Further results on robust stability of neutral system with mixed time-varying delays and nonlinear perturbations, Nonlinear Analysis, Real World Applications, 11, No. 2 (2010), 895-906.

[16] K. Ramakrishnan, G. Ray, Robust stability criteria for uncertain neutral systems with interval time-varying delay, Journal of Optimization Theory and Applications, 149, No. 2 (2011), 366-384.

[17] T. Rojsiraphisal, W. Sudsanguan, New Robust Exponential Stability Criteria for Neutral System with Mixed Constant Delays, International Journal of Pure and Applied Mathematics, (Accepted).

[18] Z. Shu, J. Lam, S. Xu, Improved estimates for neutral systems, in Asian Journal of Control, 12, No. 3 (2009), 261-270.

[19] J. Tian, L. Xiong, J. Liu, X. Xie, Novel delay-dependent robust stability criteria for uncertain neutral systems with time-varying delay, Chaos, Solitons and Fractals, 40, No. 4 (2009), 1858-1866.

[20] H. Wang, Q. Song, State estimation for neural networks with mixed interval time-varying delays, Neurocomputing, 73, No. 7-9 (2010), 1281-1288.

[21] K.W. Yu, and C.H. Lien, Stability criteria for uncertain neutral systems with interval time-varying delays, Chaos Solitons Fractals, 38, No. 3 (2008), 650-657. 